\section{Secondary care}

\section{An uphill struggle}

Sir, as the dust begins to settle and dental services are, for the most part, back up and running, I am concerned we must prepare ourselves for a continued uphill struggle in secondary care units where it seems likely there will be sustained pressure to manage the overflow of dental emergencies. As we learn to navigate the repercussions of this pandemic, the sequelae of dentists' backlog falls on the shoulders of secondary care and emergency units.

In our oral and maxillofacial unit, emergency activity has increased over $50 \%$ compared with activity one year ago, much of which consists of dental emergencies which could be more appropriately handled or prevented in primary care settings.

There is scope for reducing pressure on A\&E and emergency services with NHS 111 ensuring effective triaging processes, or indeed an increase in Urgent Dental Care centres (UDCs). If the backlog for appointments in primary care is anticipated to last over a year, I fear we will continue to experience the repercussions in secondary care long beyond that. Communication between primary and secondary care settings, UDCs and NHS 111 will be critical in the coming year to ensure dental emergencies are seen in a timely and appropriate setting.

S. Mills, Norwich, UK

https://doi.org/10.1038/s41415-021-3579-6

\section{Vocational training}

\section{Situational judgement stress}

Sir, I have often wondered about the validity of the Situational Judgement Test (SJT) as part of the national recruitment process for dental foundation training (DFT) and dental core training (DCT).

Prior to the COVID-19 pandemic, the SJT was used in combination with other methods of assessment to rank applicants. However, over the last few years, its role has varied from being the sole method of assessment to being used as a way of shortlisting.

A few questions come to mind when I reflect on my own experiences of the SJT. How does it differentiate between candidates? Is it possible to prepare for it?
Why are some people naturally better at it than others?

In my search for answers, I read with interest the article by Donnell et al. and considered the following points: attending courses does not affect SJT scores significantly and the evidence supports the validity of the SJT, albeit as part of a wider selection process. ${ }^{1}$ A systematic review and meta-analysis concluded that the SJT has moderate validity in assessing non-academic skills and predicting interpersonal performance in the workplace; however, it highlighted the difficulty of assessing these non-academic qualities.

Interestingly, the SJT best distinguishes between average and low performers, which is why it can be useful in the early stages of recruitment to select which candidates progress to the latter (often more resourceintensive) stages. ${ }^{2}$

I was most curious to find out if there is a correlation between personality type and performance in the SJT, but there is a paucity of research on this topic.

\section{R. Gorania, Sheffield, UK}

\section{References}

1. Donnell C, Walker B, Foley J. Dental foundation training in the COVID-19 era - the national recruitment lottery. Br Dent J 2021; 231: 20-25.

2. Webster E, Paton L, Crampton P, Tiffin P. Situational judgement test validity for selection: A systematic review and meta-analysis. Med Educ 2020; 54 : 888-902.

https://doi.org/10.1038/s41415-021-3577-8

\section{Coronavirus}

\section{COVID-19 'syndemic'}

Sir, there is controversy as to whether, during the pandemic, patients with chronic oral pain have found their symptoms getting better or worse. ${ }^{1,2}$ While some researchers have reported an exacerbation of symptoms in burning mouth syndrome patients, others have reported that chronic pain was not affected at all.

We have studied the impact of COVID19 on patients with oral psychosomatic disorders: burning mouth syndrome, oral cenesthopathy, atypical odontalgia, phantom bite syndrome and halitophobia, who visited our clinic (Psychosomatic Dentistry Clinic in Dental Hospital, Tokyo Medical and Dental University). Patients were divided into those from April 2018 to February 2019, deemed the pre-COVID-19 group, and those from April 2020 to February 2021, as post-COVID-19. Psychological impact was assessed using the Self-rating Depression Scale (SDS) and Pain Catastrophising Scale (PCS); however, we observed no significant differences between the two groups with respect to the mean scores of these scales.

In Japan, the government declared a state of emergency repeatedly. Although there was almost no psychological exacerbation both in our study and clinically, patients often complained about the stress they experienced. Therefore, we are now concerned that a prolonged pandemic might affect the psychological health of our patients gradually, eventually giving rise to 'syndemic' situation. This term is made up of the words 'synergy' and 'epidemic' and was first coined in 1990 by Prof M. Singer to describe when two or more diseases interact with each other to exacerbate the condition. ${ }^{3}$ Conditions of health inequality caused by social stigma, poverty, stress and so on are most likely factors responsible for a syndemic to emerge.

As with our patients, initially, many were anxious only about COVID-19 infection, but now patients complain about various psychological problems associated with COVID-19 like reduced social interaction, apprehension to make hospital visits which could deprive them from receiving the necessary treatment, work stress, financial stress for those who have lost jobs, and so forth.

These psychosocial problems could have a greater influence on dental patients and we believe might lead to deterioration of their symptoms, especially in the younger generation. Thus, we believe that more mental healthcare in dental practice is necessary, even if it is by using teledentistry.

C. Takao, G. Nayanar, A. Toyofuku, Tokyo, Japan

\section{References}

1. Mun C J, Campbell C M, McGill L S, Aaron R V. The early impact of COVID-19 on chronic pain: a crosssectional investigation of a large online sample of individuals with chronic pain in the United States, April to May, 2020. Pain Med 2021; 22: $470-480$.

2. Hudson J. How mental health affects oral health. $B D J$ Student 2021; 28: 21-23.

3. Singer M, Bulled N, Ostrach B, Mendenhall E. Syndemics and the biosocial conception of health. Lancet 2017; 389: 941-950.

https://doi.org/10.1038/s41415-021-3580-0 